\title{
Indian Subcontinent's First Proximal Forearm Level Double Upper Extremity Transplantation
}

\author{
Mohit Sharma ${ }^{1}$ Subramania lyer ${ }^{1}$ Kishore Purushottaman ${ }^{1}$ Sundeep Vijayaraghavan ${ }^{1}$ jimmy Matthew ${ }^{1}$ \\ Raghuveer Reddy Dudipala' Janarthanan Ramu Jerry Paul ${ }^{1}$ Sunil Rajan ${ }^{2}$ Anwar $^{2}$ Meera $^{2}$ Dhruvan $^{3}$ \\ Arun $^{3} \quad$ Arjun $^{3} \quad$ Vishak Verma ${ }^{1} \quad$ Ashish Chaudary ${ }^{1}$ Swapnil Dhake ${ }^{1} \quad$ Akshay Omkumar $^{1}$ \\ Radhika Kapahtia' Dimpy Sharma ${ }^{1}$ Nirav Maharaja ${ }^{1}$
}

\footnotetext{
${ }^{1}$ Department of Plastic and Reconstructive Surgery, Amrita Institute of Medical Sciences, Kochi, Kerala, India

${ }^{2}$ Department of Anaesthesiology, Amrita Institute of Medical

Sciences, Kochi, Kerala, India

${ }^{3}$ Department of Orthopedics, Amrita Institute of Medical Sciences,

Kochi, Kerala, India
}

Address for correspondence Mohit Sharma, MS, MCh, Department of Plastic and Reconstructive Surgery, Amrita Institute of Medical Sciences, Kochi 682041, Kerala, India (e-mail: ammasmohit@gmail.com).

\begin{abstract}
Keywords

- proximal forearm transplantation

- hand transplantation

- hand transplantation in Indian sub continent

- bilateral upper limb transplantation

Introduction Vascularized composite allotransplantation (VCA) has added another step to the reconstructive ladder, leading to a paradigm shift in the approach toward management of cases of upper limb amputations. In this article, we discuss in detail the technical aspects of proximal forearm level transplantation, as well as the immediate posttransplant monitoring and immunosuppression protocols.

Materials and Methods A 24-year-old male victim of an electric injury presented with a bilateral proximal forearm level amputation. After the mandatory preoperative psychiatric and immunological evaluation, the patient underwent a proximal forearm level double upper extremity transplantation. He was then put on a stringent immunosuppression and physical rehabilitative regime.

Discussion Conceptually, the proximal forearm level transplantation is significantly different from distal forearm level transplant. This transplant "reestablishes" the "donor extremity length," maintains the normal functional length of the donor muscles, and the functional return happens only after the newly transplanted donor muscles are reinnervated over a period of time.

Conclusion As the sequence of surgical repair and associated decision making could be quite confusing for this level of transplantation, it is highly advisable to do mock cadaver surgical dissections in order to standardize the procedure and make the team familiar with it.
\end{abstract}

\section{Introduction}

Vascularized composite allotransplantation (VCA) has added another step to the reconstructive ladder, leading to a paradigm shift in the approach toward management of cases of upper limb amputations. With nearly two decades ${ }^{1-3}$ of follow-up of the first cases of hand transplantation and more than satisfactory long-term outcomes, reconstructive transplantation is gaining wider acceptance. However, one needs to understand that a transplant is significantly different from a replant. ${ }^{4}$ Similarly, the approach to a transplant at a proximal forearm level is totally different to the one at distal or supracondylar level; also, the long-term rehabilitation and expected outcomes are different for a proximal forearm level transplantation. ${ }^{5,6}$ In this article, we discuss in detail the technical aspects of this complex procedure, published online

December 26, 2019
DOI https://doi.org/

$10.1055 / \mathrm{s}-0039-3401474$

ISSN 0970-0358.
C2019 Association of Plastic

Surgeons of India
License terms

(잉 (1) $\Theta \circledast$ 
as well as the immediate posttransplant monitoring, and immunosuppression protocols.

\section{Methods}

\section{Procedure}

A 24-year-old male victim of an electric injury presented with a bilateral proximal forearm level amputation.(-Fig. 1) He was on myoelectric prostheses but was not satisfied with the same. The amputation stumps were of 16 and $15 \mathrm{~cm}$ from the elbow crease on the right and the left sides, respectively. He underwent the pretransplant psychiatric evaluation as per protocol, and was also counselled about the possibility of reduction in the stump length in the event of a failure of the transplant and the possible deleterious effects of long-term immunosuppression. He was even made to have discussions with the prior hand transplant recipients to better come to terms with what to expect at different stages of the recovery.

\section{Preoperative Evaluation}

The immunological workup comprised human leucocyte antigen (HLA) typing, panel reactive antibody (PRA) and donor-specific antibody detection assays (DSA), and complement-dependent cytotoxicity assays (CDC).

He underwent a magnetic resonance imaging (MRI) scan to assess the status of the muscles and soft tissue in the stumps and evaluate the neuromas, which would help in prejudging the level of nerve repair in the patient, along with an angiogram to further assess the recipient blood vessel status.

The donor was a brain dead 29-year-old road traffic accident victim in another center, $30 \mathrm{~km}$ away.

As per our protocol for ascertaining a donor match, $\mathrm{ABO}$ compatible blood group match, and a lymphocyte cross match $<20 \%$ (preferably $<10 \%$ ) was mandatory, and this patient fulfilled these criterias and other standard criterias for donor and recipient match.

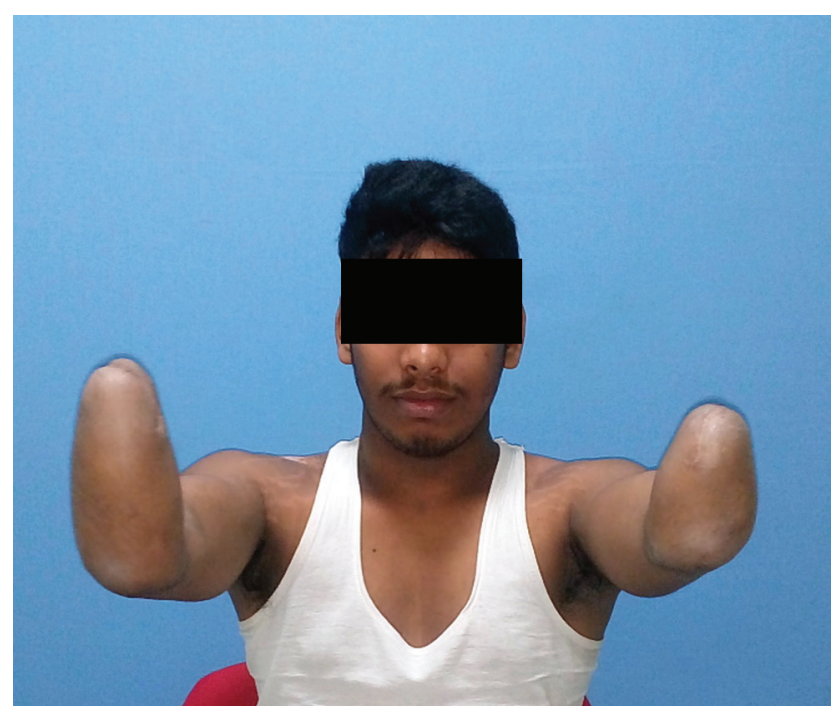

Fig. 1 Patient with bilateral proximal forearm level amputation.

\section{Surgical Technique \\ Donor Retrieval}

A tourniquet was applied over the upper arm and inflated at $100 \mathrm{~m} \mathrm{Hg}$ above the systolic blood pressure (-Figs. 2, 3). Midarm circumferential incision was given, and cephalic and basilic veins identified, ligated, cut proximally, and tagged. Biceps and brachialis muscles were transected, and median, ulnar, and radial nerves were identified, cut, and tagged. Brachial artery and venae commitantes were ligated proximally and transected. Midhumeral osteotomy was performed with an oscillating saw, and triceps muscle was transected.

The limb was retrieved and the brachial artery was perfused with University of Wisconsin solution. A note was made regarding the dominance of the superficial or deep venous system by noticing the increased flow of fluid from the particular venous system. In this patient, the deep venous system was dominant, and later while conducting the vessel anastomosis venae, commitantes were anastomosed first. This observation helps in better perfusion, minimizes the blood loss, and reduces the edema.

Sample biopsies from the donor limb were taken from skin, fat, muscle, and vessels.

As this limb was retrieved from a patient at a different center, a "Green Corridor" was estabilizhed with the help of police, while transporting the limb under the conventional Triple Bag System. The limbs were wrapped in saline sponges and covered with two sealed plastic bags which were kept in a third bag filled with ice cold saline. The third bag was also sealed for transport.

On arrival, the donor limbs were shifted to an adjacent theater to the recipient, and donor preperaton was started on a table covered with ice bags on top of which a sterile plastic sheet was placed.

\section{Donor Preparation}

The two teams which retrieved the donor hands were the ones involved in the preparation.

An incision was given midline dorsally and in the midline on flexor aspect, extending from midarm to the proximal forearm.

The dissection began by making generous skin flaps; the cephalic and basilic veins were protected and tagged, and the lateral and medial cutaneous nerves of forearm, which are adjacent to the superficial veins, were dissected and tagged individually.

The dissection continued along the medial arm. Ulnar nerve was dissected from proximal to distal, freed completely from the cubital tunnel, and distally up to the origin of the branch to the flexor carpi ulnaris.

The median nerve and brachial artery were then followed distally; the brachial artery was freed up to the divison of radial and ulnar vessels, and the median nerve was dissected up to the origin of the branch to pronator teres. The radial nerve was traced below after carefully transecting the brachioradialis muscle at its origin. The branches of radial nerve to the brachioradialis and extensor carpi radialis longus and 


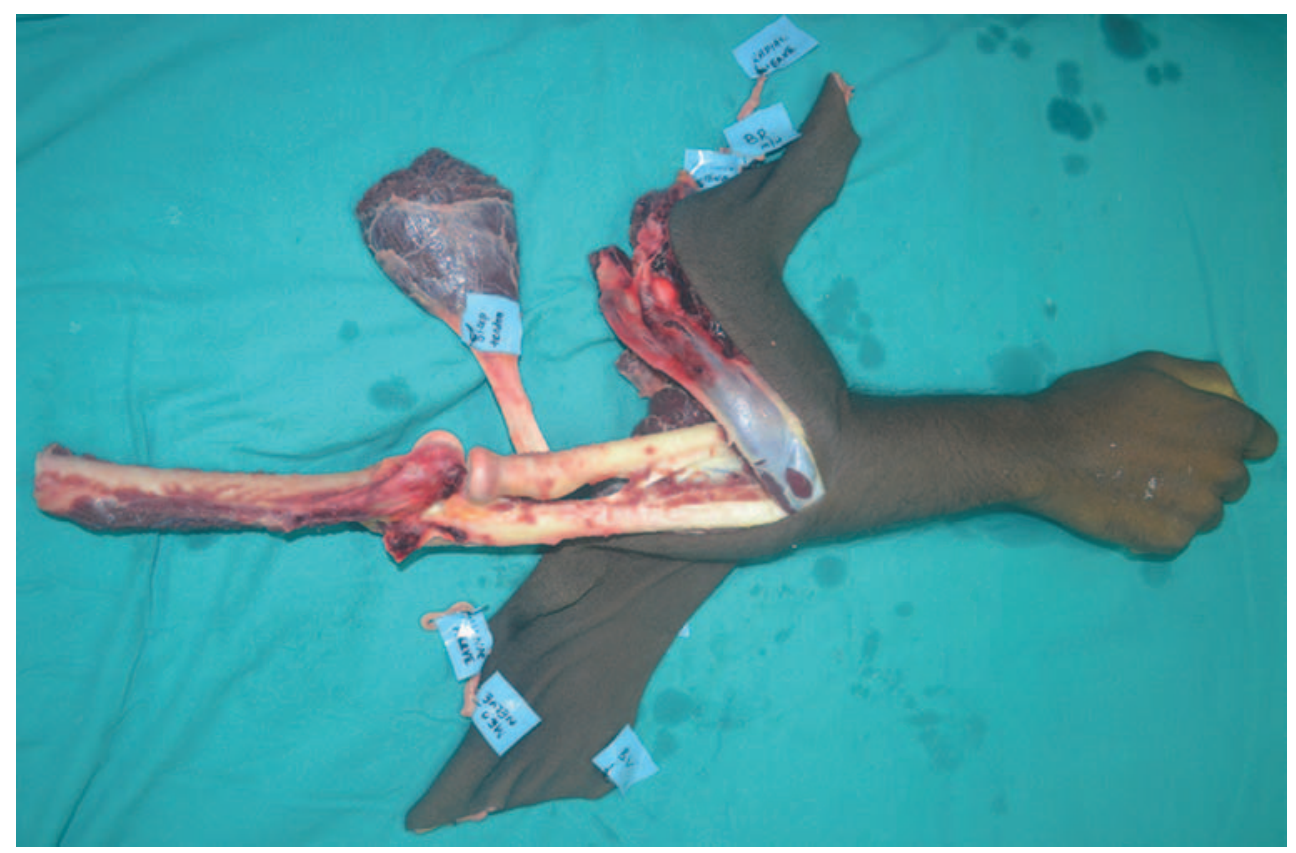

Fig. 2 Completely dissected extensor compartment structures.

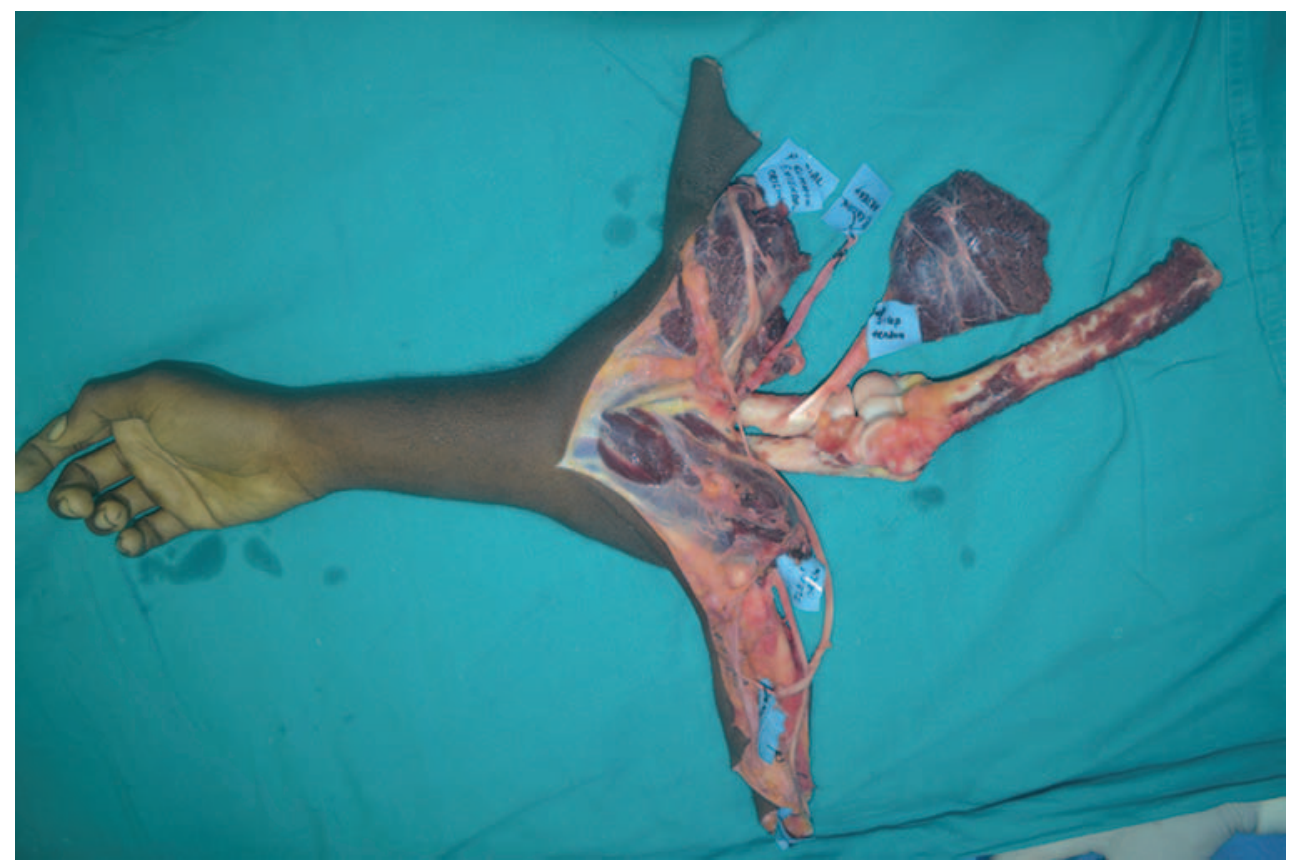

Fig. 3 Dissected flexor compartment structures.

brevis were identified, tagged, and transected at the very origin from the radial nerve for subsequent anastomosis to the recipient radial nerve branches.

The flexor and extensor origins were dissected free from the medial and lateral epicondyles, respectively, along with the attached periosteum. This helps in improving the suture purchase at the time of fixation with due care to protect the ulnar, median, and radial nerves.

The radial nerve is followed-up distally to its division into superficial and deep branches, and the deep branch was dissected till its location under the supinator muscle. Once this dissection was completed, all the nerves, vessels, and muscles of the forearm were reflected distally, with care, to avoid any stretch injury to the nerves and vessels. Special attention is required for protecting the deep branch of the radial nerve.

This reflection of all the muscles, vessels, and nerves from the radius and ulna was done keeping the interosseous membrane intact and in a subperiosteal plane.

Practically, one needs to go to almost midforearm to allow for the three holes of the limited contact dynamic compression plate (LCDCP) fixation onto the donor bones (the deep muscles, which are reflected in this fashion, do get reattached 
to the bone in time and start functioning normally when reinnervation takes place).

All the structures were individually tagged with tags created by cut Esmarch's bandage pieces and fixed by $2 / 0$ silk stitches.

The biceps tendon was followed distally to its insertion on the radius. The biceps tendon insertion is an important landmark for ascertaining the orientation of radius for preplating and osteotomy.

While carrying out the donor dissection, one needs to understand that, in the recipient, the plate will be fixed just beyond the insertion of biceps, so the recipient bone will take three holed plate after the insertion of biceps; followed by that, three holes of the plate have to be fixed on the donor bones. Therefore, for that distance, the muscles have to be reflected from the donor forearm bones.

\section{Recipient Preparation}

Midlateral skin incisions were given, and volar and dorsal skin flaps were elevated ( - Fig. 4).

The skin flaps were raised subfascially up to the distal arm but the deep fascia was incised at several places to dissect the superficial veins and cutaneous nerves off the skin flap (this dissection of nerves and superficial veins from the skin flaps was done up to the elbow crease, so that later straight line anastomosis of superficial veins could be done when the donor hands were fixed. It also helps in the trimming and adjustment of skin flaps).

All of the native flexors and extensor muscle groups were left down in place and only the median, radial, and ulnar nerves were dissected.

The radial nerve was traced distally, and its branches to the brachioradialis, extensor carpi radialis longus (ECRL), and extensor carpi radialis brevis (ECRB) were tagged individually and cut, so that later they could be repaired individually to the branches of donor muscles. Both the superficial and deep branches were isolated as distally as possible, neuromas identified and resected, and nerves tagged.

The median nerve was followed maximally down by releasing the superficial head of the pronator teres to allow for distal anastomosis.

The ulnar nerve was prepared to the most distal end and transposed anteriorly to shorten the recovery time and avoid later stretch-induced neuropathy.

The brachial artery was isolated just before its division in to the radial and ulnar arteries, and prepared for end-to-end anastomosis with the donor brachial vessel.

The radius and ulna were exposed and subperiosteal dissection was performed to reflect both flexor and extensor muscle groups from the bones. The upper limit of dissection was the distal end of insertion of the biceps tendon. The osteopenic bone was removed from the free ends. but the periosteum was reflected and preserved.

The recipient bones were preplated with 3.5-mm LCDCPs, incorporating three holes on each bone. On the radius, the plate was fixed dorsolaterally, and on the ulna, it was fixed dorsomedially (one important aspect of preplating is to countour the plates before hand according to the curvature of radius and ulna. This step helps us to maintain the normal anatomical gap between the radius and ulna, facilitating normal supination and pronation. If the plates remain straight, the bones would come near and supination and pronation movement would be restricted).

\section{Recipient Operation}

One important aspect of the operation is to achieve the correct extremity length, and in the proximal forearm-level transplant, the final length of extremity achieved is determined by the length of the donor limb. To ascertain the length of bone to be discarded, the donor limb was brought to match with the recipient limb, and lateral epicondyle of donor limb humerus was matched to the lateral epicondyle of the recipient humerus ( - Fig. 5). The donor bones were marked to the

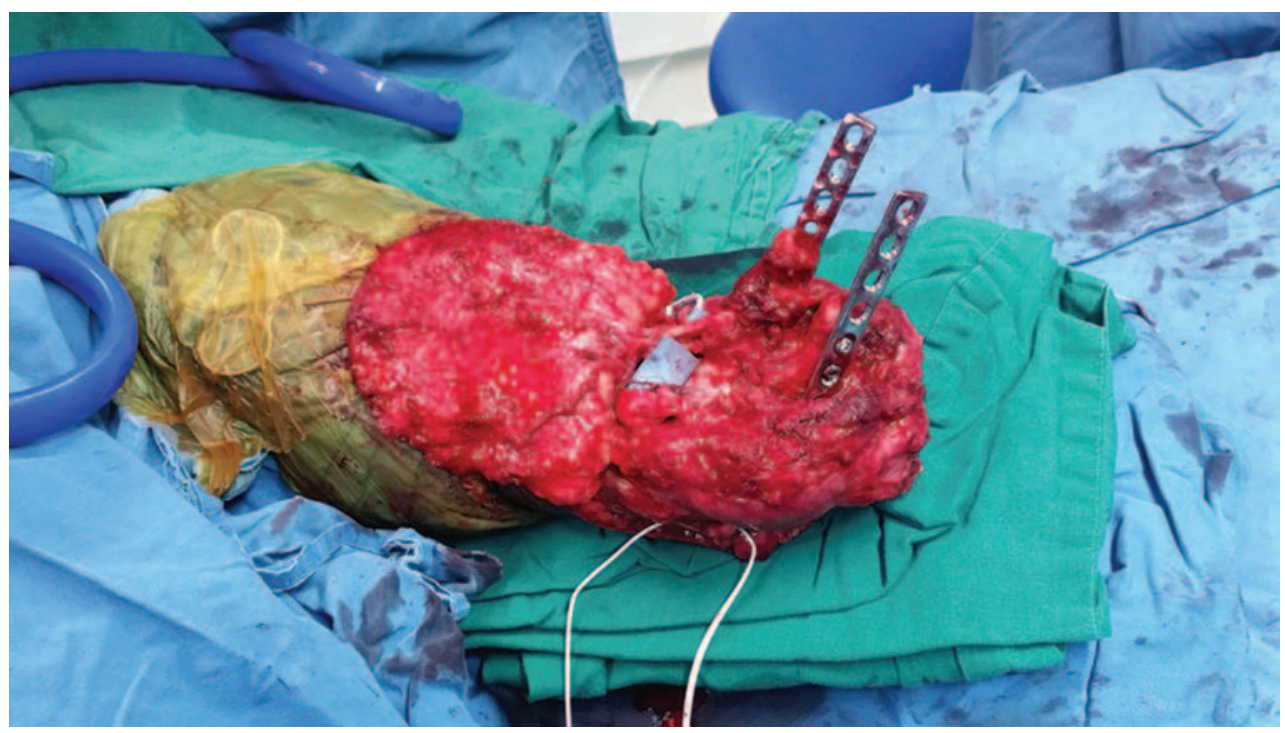

Fig. 4 Pre-plated recipient radius and ulna. 


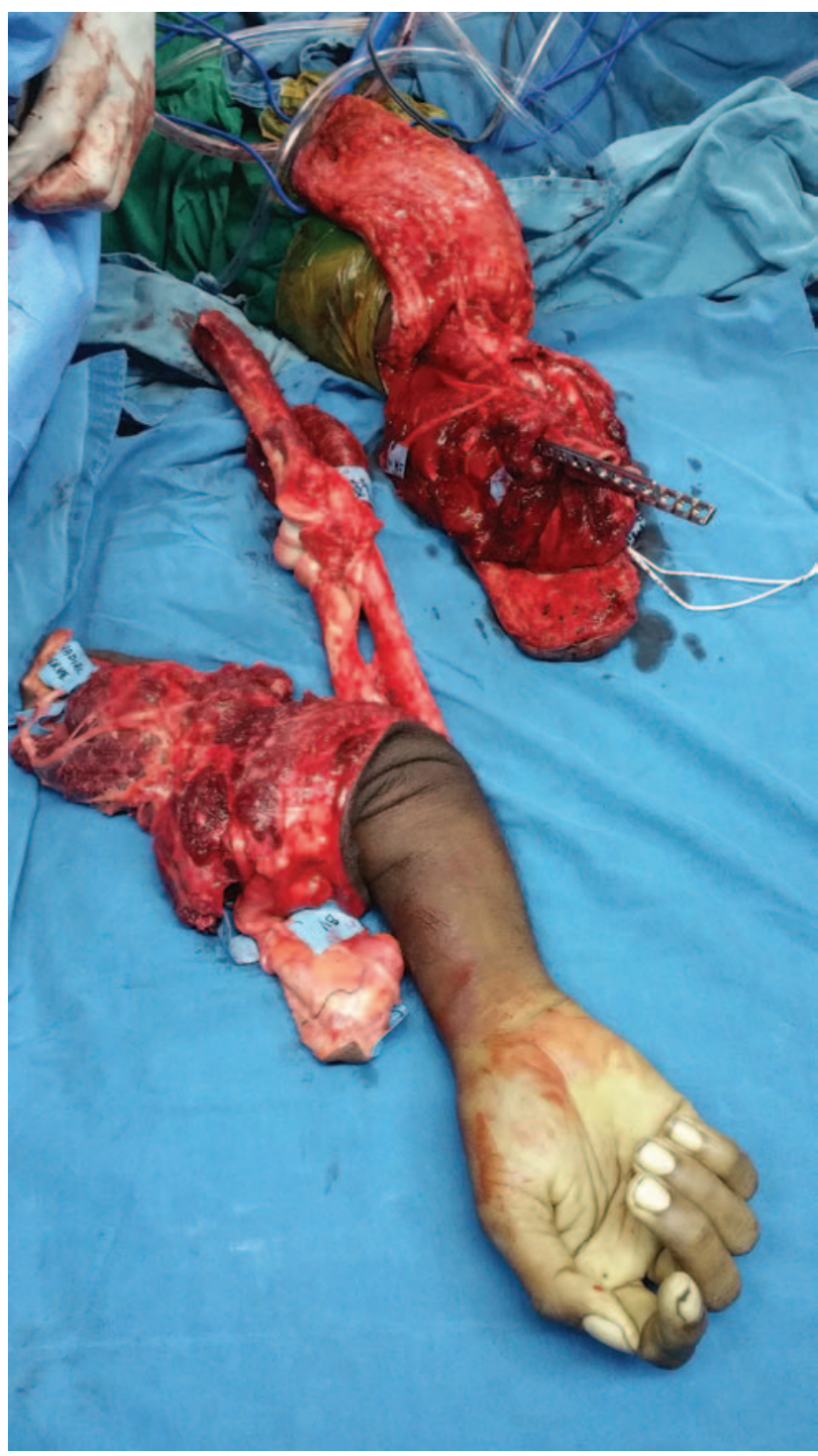

Fig. 5 Donor and Recipient extremities brought together.

available length of the recipient bones; accordingly, only that much bone was discarded from the donor limb depending on the availability in the recipient ( - Figs. 6, 7). Before discarding the donor bone, the periosteal flaps were reflected. (Bicipital tuberosity should be facing medially in full supination, and that is used as a guide for the correct orientation and alignment of radius and ulna at the time of osteosynthesis)

Once these parameters were satisfied, osteosynthesis was performed with screws traversing both the cortices, and the reflected periosteal flaps were kept back on the bone (this step facilitates the union of plated bones by allowing for the neoosteogenisis).

Immediately thereafter, vessel anastomosis was performed (-Fig. 8).

The brachial artery was anastomosed end-to-end with $8 / 0$ nylon.

In this patient, as previously noted, the deep venous system was dominant; therefore, the venae commitantes were anastomosed first. This minimized the blood loss and prevented excessive tissue edema.
The deep branch of radial nerve was anastomosed epineurally using 9/0 Nylon as distally as possible. This was done before attaching the combined extensor origin to the lateral epicondyle of the recipient humerus.

In this technique, the donor muscle group is effectively overlapped over the denervated recipient muscles, and some portion of recipient muscle mass must be removed to create the space for attachement of donor muscles.

Similarly, the common flexor origin was attached to the medial epicondyle.

In both medial and lateral epicondyles, a hole was created with 2-mm drill bit, and number-1 polypropylene suture was used to fix the common extensor and flexor origins to the bone using multiple passes through the thick harvested periosteum.

The donor brachioradialis, ECRL, and ECRB were overlapped over the partially debulked native brachioradialis, ECRL, and ECRB, and sutured using the 3-0 polyglactin-interrupted sutures. Brachioradialis was also secured to the lateral supracondylar ridge of the humerus using 2-0 polypropylene suture. The individual nerve branches of brachioradialis, ECRL, and ECRB were anastomosed to the recipient branches using 10-0 Nylon epineural stitches and secured with Evicel (Johnson and Johnson) glue.

The anteriorly transposed ulnar nerve was anastomosed using 9-0 Nylon epineural stitches along with Evicel glue. The median nerve was repaired before the origin of the anterior interosseus branch.

The lateral and medial cutaneous nerves of forearm were anastomosed using 10-0 Nylon and Evicel glue.

Finally, the cephalic and basilic veins were anastomosed using 9-0 Nylon in as straight a line as possible, This reduces the possibility of kinking in the postoperative period. This was followed by placement of suction drains and closure of skin flaps.

\section{Dorsal and Volar Suction Drains}

The skin flaps were approximated in a zigzag fashion to avoid a constricting closure. Excess flaps were trimmed and layered closure was performed (-Figs. 9, 10).

\section{Immunosuppression and Monitoring}

\section{Induction and Maintenance Regime}

On confirmation of a favorable lymphocyte cross match of less than $10 \%$, the recipient was started on an induction immune-suppression regime of injection thymoglobulin at $1.5 \mathrm{mg} / \mathrm{kg}$, injection methyl prednisolone of $500 \mathrm{mg}$, capsule tacrolimus at $0.05 \mathrm{mg} / \mathrm{kg}$, and tablet mycophenolate mofetil of $1,000 \mathrm{mg}$.

Maintainence was conducted by standard triple drug regime using prednisolone, tacrolimus, and mycophenolate mofetil.

\section{Monitoring Protocol}

Monitoring was carried out by skin biopsies using a 4-mm punch every week for the first 3 months, every 2 weeks for 6 months, and monthly thereafter up till 1 year. Thereafter, any time on suspicion of any rejection episode, the assessment was done using the Banff criteria. $^{7}$ 


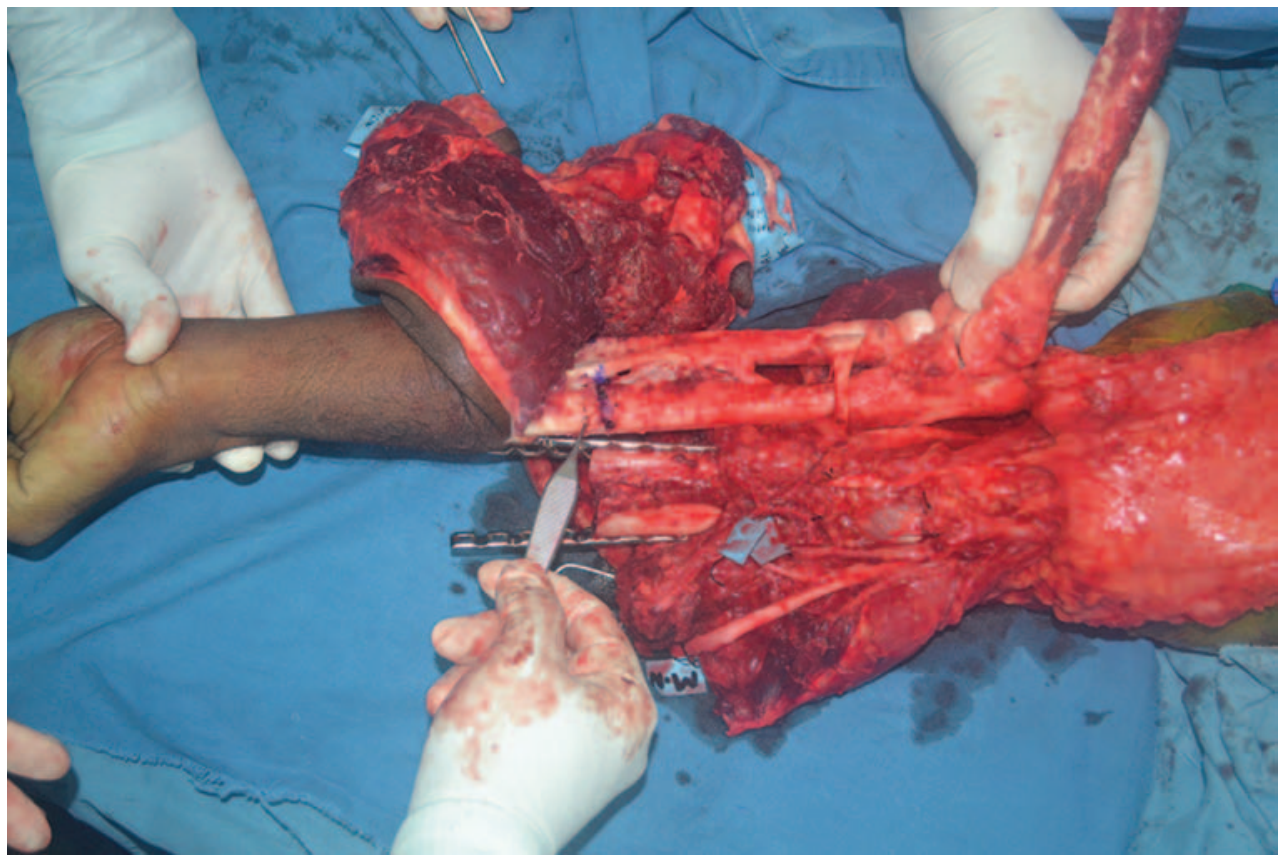

Fig. 6 Matching of the lateral epicondyles and marking of the bones to be discarded from the donor extremity.

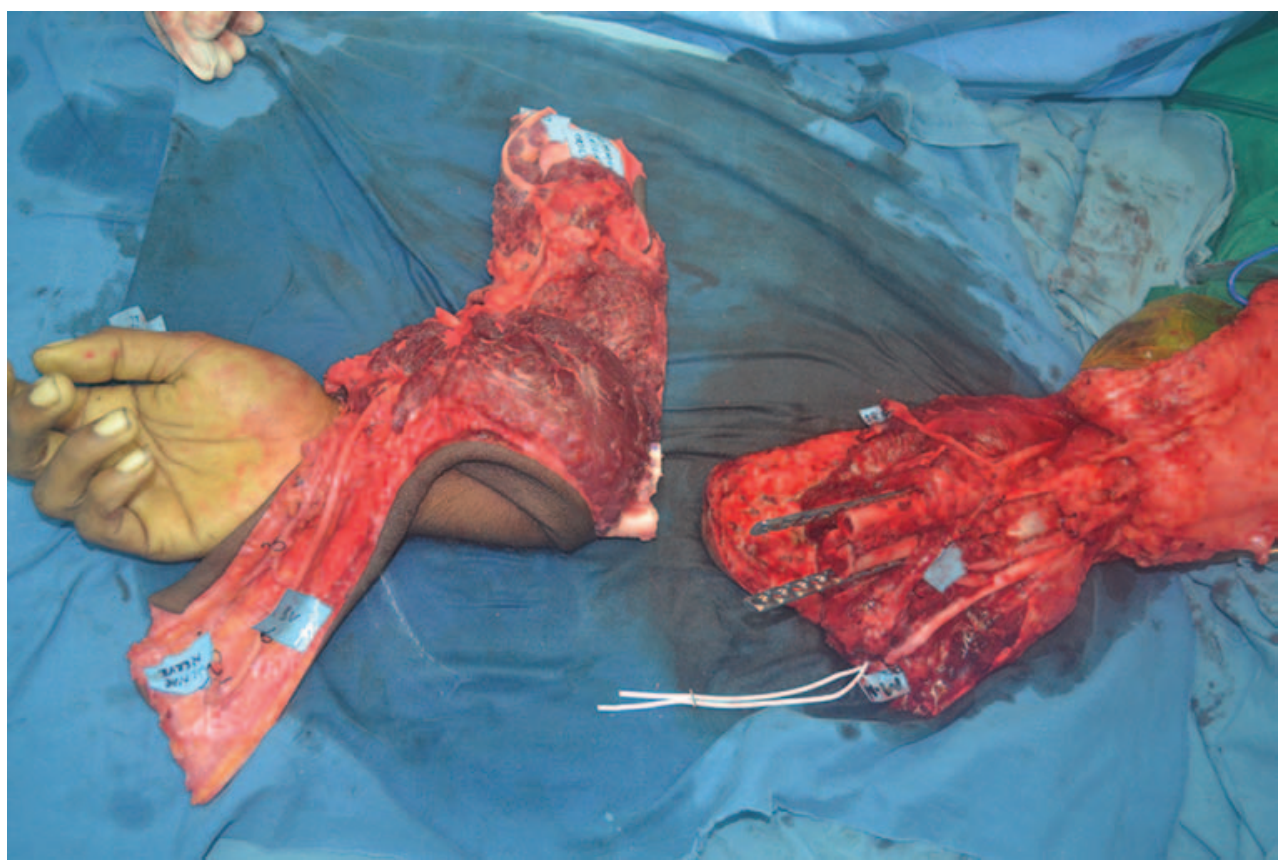

Fig. 7 Donor limb after bone removal.

Systemic levels of tacrolimus were assessed every week for the first 6 weeks, every alternate week for the next 6 weeks, and thereafter monthly assessment was continued, keeping the target tacrolimus level between 5 and $10 \mathrm{ng} / \mathrm{dL}$.

\section{Immediate Postoperative Monitoring and Care}

The patient was kept in the intensive care unit (ICU) for 2 weeks and thereafter shifted to the transplant ward. Standard isolation precautions were followed. The vascularity and vital signs were checked regularly with pulse oximeter, blood counts, serum creatinine, blood glusoce, and lipid profile being monitored regularly.

\section{Discussion}

Conceptually, the proximal forearm level transplantation is significantly different from distal forearm level transplant. $^{5}$ 


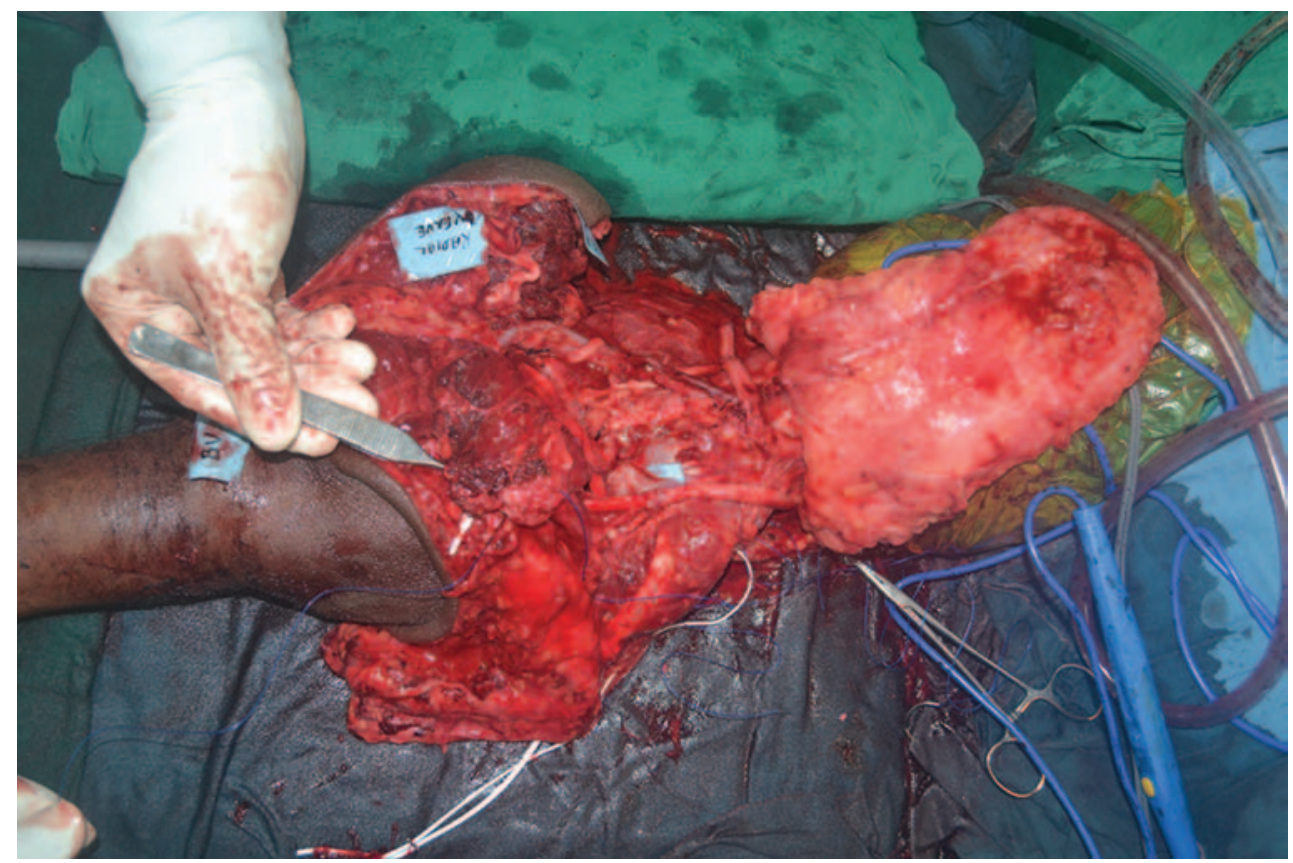

Fig. 8 Transplanted extremity after osteosynthesis and vascular anastomosis.

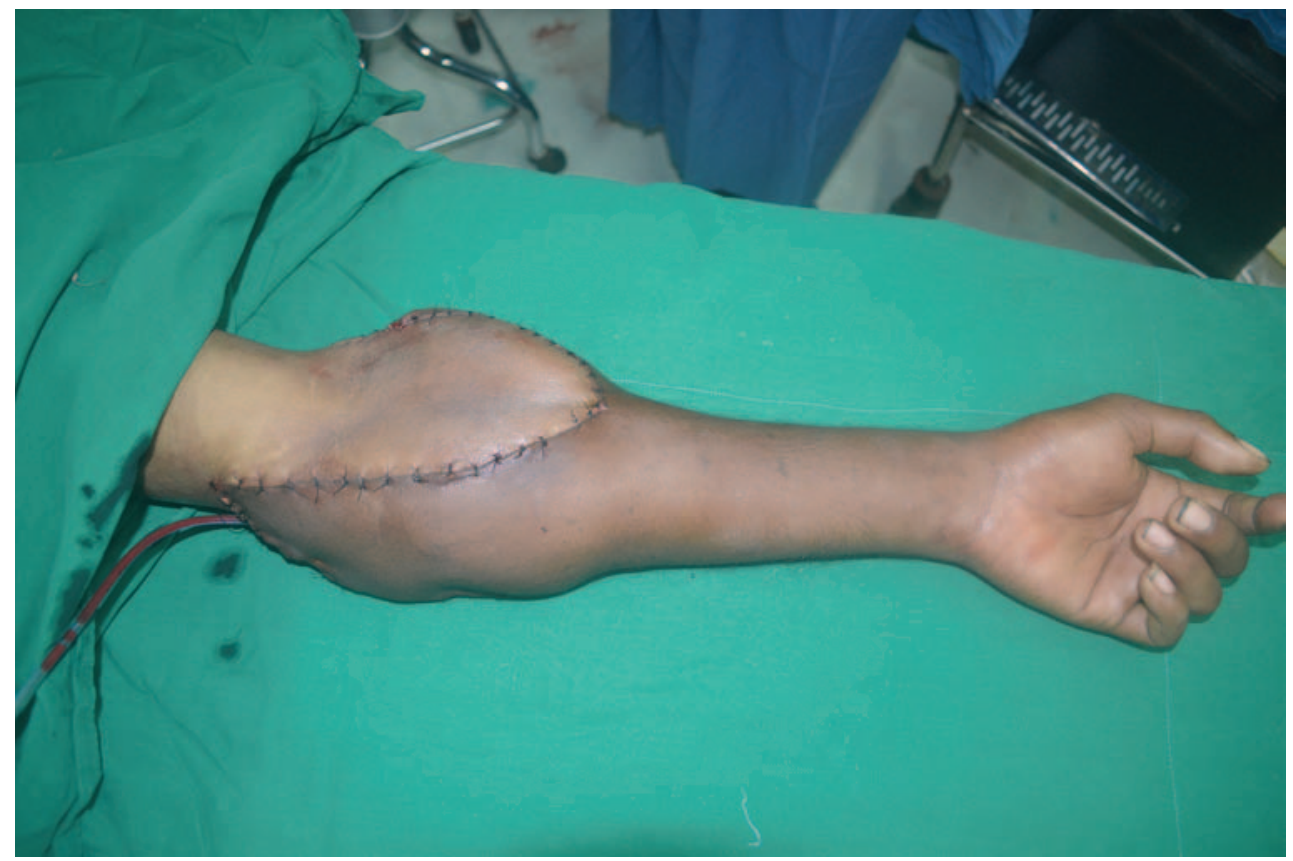

Fig. 9 Completed transplantation of left side.

In distal level transplant, the motors are already present, immediate movement is possible, and the recipient extremity length is reestablished. The number of structures needed to be sutured is significantly higher and ischemia time is less of a concern because the muscle mass is significantly less.

In the proximal forearm level transplant, usually only half or one-third of the muscle mass is present, which may not be enough to give sufficient excursion to the digits if end-to-end approximation is performed. Therefore, first the recipient muscle groups are denerveated by deligently dissecting out the radial, median, and ulnar nerves. Followed by this, some of the denervated recipient mucle bulk is discarded. This helps in accommodating the donor muscle overlap over the recipient muscles.

Preservation of some recipient muscles is potentially helpful in case the graft fails.In such a scenario the stumps would still be graftable and the level of amputation stump would not change

This transplant "reestablishes" the "donor extremity length," maintains the normal functional length of the donor muscles, and the functional return happens only after the 


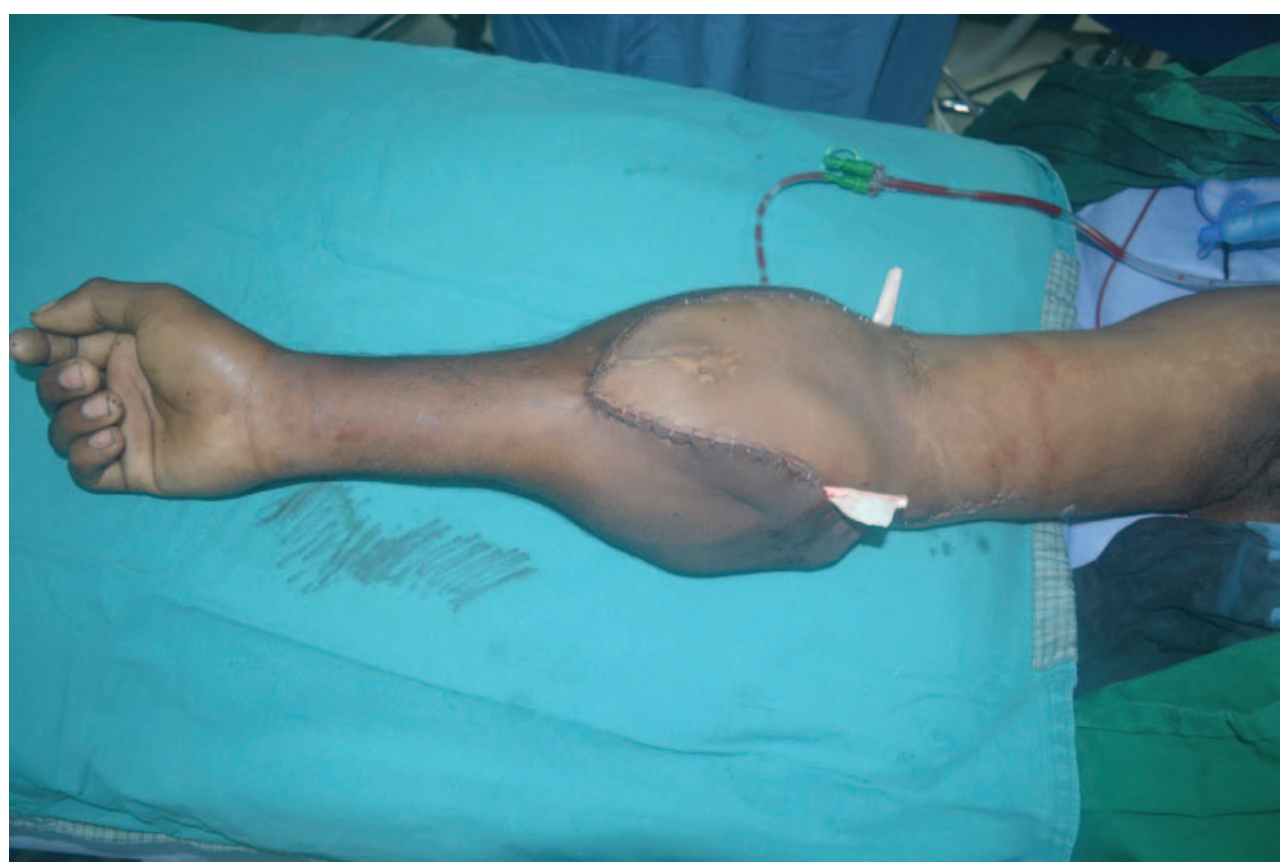

Fig. 10 Completed transplantation of right side.

newly transplanted donor muscles are reinnervated over a period of time.

In this situation, the rehabilitation period is much longer and functional return of intrinsic muscles is not as efficient as it is in a distal-level transplant.

The feasibility of this transplant depends on the length of bone available below the level of biceps tendon insertion; this bone should be able to accommodate at least three holes of the 3.5-mm LCDCP.

If enough bone is not available below the elbow, one would need to go at the supracondylar level to perform the transplant, which has an inherent risk if there is graft failure.

\section{Conclusion}

As the sequence of surgical repair and associated decision-making could be quite confusing for this level of transplantation, it is highly advisable to perfrom mock cadaver surgical dissections in order to standardize the procedure and make the team familiar with it.

In this paper, we have attempted to emphasize the rationale behind each surgical step which could be useful for the newer transplant teams.

\section{Conflict of Interest}

None.

\section{Acknowledgment}

We, the authors thank Dr. Jaimie Shores, Associate professor Orthopedic and Plastic surgery, Johns Hopkins university school of medicine, for helping us with the technical details and know-how of this transplantation.

\section{References}

1 Foroohar A, Elliott RM, Kim TWB, Breidenbach W, Shaked A, Levin LS. The history and evolution of hand transplantation. Hand Clin 2011;27(4):405-409, vii

2 Dubernard JM, Owen E, Herzberg G, et al. Human hand allograft: report on first 6 months. Lancet 1999;353(9161):1315-1320

3 Jones JW, Gruber SA, Barker JH, Breidenbach WC; Louisville Hand Transplant Team. Successful hand transplantation. One-year follow-up. N Engl J Med 2000;343(7):468-473

4 Azari KK, Imbriglia JE, Goitz RJ, et al. Technical aspects of the recipient operation in hand transplantation. J Reconstr Microsurg 2012;28(1):27-34

5 McClelland B, McCabe S, Haykal S, Borschel GH, Zuker RM, Levin LS. Proximal forearm transplantation for below elbow amputations: rationale and surgical technique. Vascularized Composite Allotransplantation 2015;2(1):26-28

6 Iglesias M, Hernandez FS, Ramirez-Berumen MF, et al. Anatomical and microsurgical implications in total and mid arm transplantation. J Reconstr Microsurg Open 2017;2:e94-e102

7 Cendales LC, Kanitakis J, Schneeberger S, et al. The Banff 2007 working classification of skin-containing composite tissue allograft pathology. Am J Transplant 2008;8(7):1396-1400 